\title{
Effect of Axial Magnetic Field on Demixing of a Binary Fluid Mixture Due to the Rotation of a Heated Sphere
}

\author{
B. R. Sharma \\ Department of Mathematics \\ Dibrugarh University-786004
}

\author{
Kabita Nath \\ Department of Mathematics \\ Dibrugarh University-786004
}

\begin{abstract}
The paper presents numerical investigation of effects of pressure gradient and temperature gradient on demixing of a binary mixture of thermally and electrically conducting incompressible viscous fluids set in motion due to the rotation of a heated sphere in presence of a uniform magnetic field applied in a direction parallel to the axis of rotation. The boundary layer equations governing the motion, temperature and concentration distribution are solved numerically with MATLAB's built - in solver bvp4c. The numerical data is represented graphically and the effects of various parameters on demixing of the binary fluid mixture are discussed.
\end{abstract}

Keywords: pressure gradient, temperature gradient, magnetic field, binary fluid mixture.

\section{INTRODUCTION}

Researchers in engineering and scientific field have shown great interest in the study of demixing of binary fluid mixtures due to its importance in industrial processes. Much interest has been attached to the process of demixing wherein one of the components of a binary mixture of viscous incompressible fluids is present in extremely small proportion. The demixing of isotopes in its naturally occurring mixture is one such example. Another example is the process of demixing of gases in the air by the pressure and temperature gradient present in the atmosphere. If one of the components is present in a small quantity then the density and the velocity of the mixture will be independent of the distribution of the components. The flow of a binary mixture is then identical to that of a single fluid but the density and the velocity are understood as the mass average density and velocity respectively i.e.,

$$
\begin{aligned}
& \rho=\rho_{1}+\rho_{2}, \\
& \vec{v}=\left(\rho_{1} \overrightarrow{v_{1}}+\rho_{2} \overrightarrow{v_{2}}\right) / \rho,
\end{aligned}
$$

where subscripts 1 and 2 respectively denote the rarer and more abundant components of the mixture. It is assumed that the first component of the binary fluid mixture is lighter than the second one. The composition of binary mixture is described by the concentration $c_{1}$, defined as the ratio of mass of rarer and lighter component to the total mass of the mixture in a given volume. The concentration $c_{2}$ of heavier and abundant component is given by $c_{2}=1-$ $c_{1}$. A binary mixture subject to the temperature gradient can generate thermal diffusion i.e. the temperature gradients cause solute fluxes. This phenomenon is known as Soret effect. The diffusion flux $\vec{\imath}$ is given by

$\vec{\imath}=-\rho D\left[\nabla \mathrm{c}_{1}+\mathrm{k}_{\mathrm{p}} \nabla \mathrm{p}+\mathrm{k}_{\mathrm{T}} \nabla \mathrm{T}\right]$, where $\mathrm{D}$ is the diffusion coefficient, $\mathrm{k}_{\mathrm{p}} \mathrm{D}$ is the barodiffusion coefficient and $\mathrm{k}_{\mathrm{T}} \mathrm{D}$ is the thermal diffusion coefficient.

Howarth [1], Nigam [2] and Banks [3] have discussed the flow of an incompressible viscous fluid due to the rotation of a solid sphere. Singh [4] and Banks [5] have solved the problem of heat transfer in the flow of an incompressible viscous fluid due to steady rotation of a uniformly heated sphere. Kalita [6] has discussed this problem under the effect of a magnetic field and has integrated the equation by Kărmăn Polhausen method. Liu [7] has discussed the flow of a binary mixture of gases of unequal molecular weights over the surface of a heated wall and has shown that the demixing of two components of the mixture takes place due to the temperature gradient existing in the thermal boundary layer along the surface of the wall. Sharma and Singh [8,9,10], Sharma and Nath [11] and Sharma et al. [12, 13] have studied the effect of magnetic field on demixing of a binary fluid mixture. Sharma and Singh [14, 15] have studied the effect of temperature gradient on demixing of species in hydromagnetic flow of a binary mixture of incompressible viscous fluids between two parallel plates, first taking the plates horizontal and second by taking the plates vertical. They found that the effect of temperature gradient is to separate the components of the binary mixture and the magnetic field increases the effect of species demixing

In this piece of work the mass transfer in a binary mixture of thermally and electrically conducting incompressible viscous fluids set in steady laminar motion due to the uniform rotation of a sphere about a diameter is studied numerically considering that the fluid at infinity is at rest. The solutions of the boundary layer equations have been obtained by expanding the functions in ascending powers of $\sin \theta$, where $\theta$ is the angle made at the centre of the sphere by the position vector of a point with the direction of the axis of rotation.

\section{FORMULATION OF THE PROBLEM}

Consider the phenomenon of species demixing due to pressure gradient and temperature gradient in a hydromagnetic flow of a binary mixture of an electrically conducting incompressible Newtonian fluids due to the rotation of a sphere $\mathrm{r}=$ a rotating with a constant angular velocity $\Omega$ about one of its diameters. The surface of the sphere is maintained at a constant temperature $T_{w}$ which is higher than $\mathrm{T}_{\infty}$, the temperature of the ambient fluid mixture at a large distance from the surface of the sphere. 
$\operatorname{Let}\left(v_{r}, v_{\theta}, v_{\phi}\right)$ be the components of velocity $\vec{v}$ in spherical polar co-ordinate system $(r, \theta, \phi)$. A weak magnetic field of strength $B_{0}$ is applied in a direction parallel to the axis of rotation. Since the motion is axisymmetric, all the physical quantities will be independent of $\phi$. Let $\mathrm{T}$ and $\mathrm{p}$ denote the temperature and the pressure respectively at any point in the fluid mixture.

Under boundary layer approximations, the equation of continuity is

$$
\frac{\partial v_{r}}{\partial r}+\frac{1}{a} \frac{\partial v_{\theta}}{\partial \theta}+\frac{v_{\theta}}{a} \cot \theta=0,
$$

and the equations of motion in directions of $r, \theta, \phi$ respectively can be written as:

$-\frac{1}{a}\left(v_{\theta}^{2}+v_{\phi}^{2}\right)=-\frac{1}{\rho} \frac{\partial p}{\partial r}-\frac{\sigma B_{0}^{2}}{\rho} v_{\theta} \sin \theta \cos \theta$

$v_{r} \frac{\partial v_{\theta}}{\partial r}+\frac{v_{\theta}}{a} \frac{\partial v_{\theta}}{\partial \theta}-\frac{v_{\phi}^{2}}{a} \cot \theta=v \frac{\partial^{2} v_{\theta}}{\partial r^{2}}-\frac{\sigma B_{0}^{2}}{\rho} v_{\theta} \cos ^{2} \theta$,

$v_{r} \frac{\partial v_{\phi}}{\partial r}+\frac{v_{\theta}}{a} \frac{\partial v_{\phi}}{\partial \theta}+\frac{v_{\theta} v_{\phi}}{a} \cot \theta=v \frac{\partial^{2} v_{\phi}}{\partial r^{2}}-\frac{\sigma B_{0}^{2}}{\rho} v_{\phi}$,

where $v$ is the coefficient of kinematic viscosity and $\sigma$ is the electrical conductivity of the medium.

The equation governing the temperature is

$$
\begin{aligned}
& c_{v}\left(v_{r} \frac{\partial T}{\partial r}+\frac{v_{\theta}}{a} \frac{\partial T}{\partial \theta}\right)=\frac{k}{\rho} \frac{\partial^{2} T}{\partial r^{2}}+v\left[\left(\frac{\partial v_{\theta}}{\partial r}\right)^{2}+\left(\frac{\partial v_{\phi}}{\partial r}\right)^{2}\right]+ \\
& \frac{\sigma B_{0}^{2}}{\rho}\left[v_{\theta}^{2} \cos ^{2} \theta+v_{\phi}^{2}\right],
\end{aligned}
$$

where $c_{v}$ is the specific heat at constant volume and $\mathrm{k}$ is the coefficient of thermal conductivity of the binary mixture.

The equation for the concentration ratio $c_{1}$ in spherical polar co-ordinates with boundary layer approximations is given by

$\rho\left(v_{r} \frac{\partial c_{1}}{\partial r}+\frac{v_{\theta}}{a} \frac{\partial c_{1}}{\partial \theta}\right)=\rho D\left[\begin{array}{c}\frac{\partial^{2} c_{1}}{\partial r^{2}}+\frac{\partial}{\partial r}\left(k_{p} \frac{\partial p}{\partial r}\right) \\ +\frac{\partial}{\partial r}\left(k_{T} \frac{\partial T}{\partial r}\right)\end{array}\right]$.

The coefficients $k_{p}$ and $k_{T}$ may be determined from the thermodynamic properties alone. Landau and Lifshitz [16] have given the explicit expression for the barodiffusion ratio $k_{p}$ as

$k_{p}=\left(m_{2}-m_{1}\right)\left[\frac{c_{1}}{m_{1}}+\frac{c_{2}}{m_{2}}\right] \frac{c_{1} c_{2}}{p_{\infty}}$,

since $c_{2}=1-c_{1}$ and if $c_{1}$ is assumed to be very small so that $c_{1}^{2}$ may be neglected then (10) becomes

$k_{p}=\left(m_{2}-m_{1}\right) \frac{c_{1} c_{2}}{p_{\infty}}=A c_{1}$,

where $\mathrm{A}=\frac{\left(m_{2}-m_{1}\right)}{m_{2} p_{\infty}}$.

The expression for $k_{T}$ has been suggested by Hurle and Jakeman [17] as

$k_{T}=S_{T} c_{1} c_{2}$

for small value of $c_{1}$ (13) becomes

$k_{T}=S_{T} c_{1}$.

The boundary conditions of the problem are $v_{r}=v_{\theta}=0, v_{\phi}=a \Omega \sin \theta, T=T_{w}, \rho c_{1} v_{r}-$ $\left.\rho D\left(\frac{\partial c_{1}}{\partial r}+A c_{1} \frac{\partial p}{\partial r}+S_{T} c_{1} \frac{\partial T}{\partial r}\right)+\beta \sigma v_{\phi} B_{0} \sin \theta=0\right\}$

where $\beta$ is the electrical characteristic of the conducting medium and is assumed to be constant here.

And

$v_{\theta} \rightarrow 0, v_{\phi} \rightarrow 0, T \rightarrow T_{\infty}, c_{1} \rightarrow\left(c_{1}\right)_{\infty} \quad$ as $r \rightarrow \infty(15)$ where $\left(c_{1}\right)_{\infty}$ be the fixed value of the concentration for the rarer and lighter species in its undisturbed state at infinity.

\section{SOLUTION OF THE PROBLEM}

For the flow problem $v_{r}, v_{\theta}, v_{\phi}, p, T$ and $c_{1}$ are expanded in powers of $\sin ^{2} \theta$ as

$$
\begin{aligned}
& v_{r}=-\sqrt{\nu \Omega}\left[2 F_{1}+\left(4 F_{3}-3 F_{1}\right) \sin ^{2} \theta+\left(6 F_{5}-\right.\right. \\
& \left.\left.5 F_{3}\right) \sin ^{4} \theta+\cdots\right] \\
& v_{\theta}=\frac{a \Omega}{2} \sin 2 \theta\left[F_{1}^{\prime}+F_{3}^{\prime} \sin ^{2} \theta+F_{5}^{\prime} \sin ^{4} \theta+\cdots\right] \\
& v_{\phi}=a \Omega \sin \theta\left[G_{1}+G_{3} \sin ^{2} \theta+G_{5} \sin ^{4} \theta+\cdots\right] \\
& p=\rho a \Omega^{2} \sqrt{\frac{v}{\Omega}}\left[p_{0}+p_{1} \sin ^{2} \theta+p_{3} \sin ^{4} \theta+\cdots\right] \\
& T=T_{\infty}+\frac{a^{2} \Omega^{2}}{c_{v}}\left[T_{1}+T_{3} \sin ^{2} \theta+T_{5} \sin ^{4} \theta+\cdots\right] \\
& c_{1}=\left(c_{1}\right)_{\infty}\left[\psi_{0}+\psi_{1} \sin ^{2} \theta+\psi_{3} \sin ^{4} \theta+\cdots\right]
\end{aligned}
$$

where $F_{i}, G_{i}, p_{i}, T_{i}, \psi_{i}(\mathrm{i}=0,1,2,3, \ldots)$ are functions of the independent non-dimensional variable $\eta$ given by

$\eta=\sqrt{\frac{\Omega}{v}}(r-a)$.

With the above assumptions the equation of continuity (4) is satisfied identically.

On substituting the expansions (16) to (21) in the equations (5) to (9) and equating the like powers of $\sin \theta$ and $\sin ^{2} \theta$ on both sides of the equations the following non-linear coupled ordinary differential equations are obtained:

$$
\begin{aligned}
& F_{1}^{\prime 2}-G_{1}^{2}-2 F_{1} F_{1}^{\prime \prime}+N F_{1}^{\prime}=F_{1}^{\prime \prime \prime} \\
& 4 F_{1}^{\prime} F_{3}^{\prime}-2 F_{1} F_{3}^{\prime \prime}-4 F_{3} F_{1}^{\prime \prime}+3 F_{1} F_{1}^{\prime \prime}-2{F_{1}^{\prime}}^{2}-2 G_{1} G_{3}+ \\
& N\left(F_{3}^{\prime}-F_{1}^{\prime}\right)=F_{3}^{\prime \prime \prime} \\
& p_{0}^{\prime}=0 \\
& F_{1}^{\prime 2}+G_{1}^{2}-N F_{1}^{\prime}=p_{1}^{\prime} \\
& 2 F_{1}^{\prime} F_{3}^{\prime}-F_{1}^{\prime 2}+2 G_{1} G_{3}-N\left(F_{3}^{\prime}-F_{1}^{\prime}\right)=p_{3}^{\prime} \\
& 2 F_{1}^{\prime} G_{1}-2 F_{1} G_{1}^{\prime}+N G_{1}=G_{1}^{\prime \prime} \\
& 4 F_{1}^{\prime} G_{3}+2 F_{3}^{\prime} G_{1}-2 F_{1}^{\prime} G_{1}-4 F_{3} G_{1}^{\prime}+3 F_{1} G_{1}^{\prime}-2 F_{1} G_{3}^{\prime}+ \\
& N G_{3}=G_{3}^{\prime \prime} \\
& T_{1}^{\prime \prime}+2 P_{r} F_{1} T_{1}^{\prime}=0 \\
& P_{r}\left[2 F_{1}^{\prime} T_{3}-2 F_{1} T_{3}^{\prime}++3 F_{1} T_{1}^{\prime}-4 F_{3} T_{1}^{\prime}-N\left(F_{1}^{\prime 2}+G_{1}^{2}\right)\right]= \\
& T_{3}^{\prime \prime}+P_{r}\left(F_{1}^{\prime \prime 2}+G_{1}^{\prime 2}\right) \\
& \psi_{0}^{\prime \prime}+2 S_{c} F_{1} \psi_{0}^{\prime}+B_{d}\left(\psi_{0} p_{0}^{\prime \prime}+\psi_{0}^{\prime} p_{0}^{\prime}\right)+t_{d}\left(\psi_{0} T_{1}^{\prime \prime}+\right. \\
& \left.\psi_{0}^{\prime} T_{1}^{\prime}\right)=0 \\
& (32) \\
& \psi_{1}^{\prime \prime}+S_{c}\left(4 F_{3} \psi_{0}^{\prime}-3 F_{1} \psi_{0}^{\prime}+2 F_{1} \psi_{1}^{\prime}-2 F_{1}^{\prime} \psi_{1}\right)+ \\
& B_{d}\left(\psi_{0} p_{1}^{\prime \prime}+\psi_{1} p_{0}^{\prime \prime}+\psi_{0}^{\prime} p_{1}^{\prime}+\psi_{1}^{\prime} p_{0}^{\prime}\right)+t_{d}\left(\psi_{0} T_{3}^{\prime \prime}+\psi_{1} T_{1}^{\prime \prime}+\right. \\
& \left.\psi_{0}^{\prime} T_{3}^{\prime}+\psi_{1}^{\prime} T_{1}^{\prime}\right)=0
\end{aligned}
$$

where $N=\frac{\sigma B_{0}^{2}}{(\rho \Omega)} \quad$ is the hydro-magnetic interaction parameter, $P_{r}=\frac{\rho v c_{v}}{k}$ is the Prandtl number, $S_{c}=\frac{v}{D}$ is the Schmidt number, $B_{d}=\left(A \rho a \Omega^{2} \sqrt{\frac{v}{\Omega}}\right)$ is the barodiffusion number and $t_{d}=\frac{S_{T} a^{2} \Omega^{2}}{c_{v}}$ is the thermal diffusion number. The boundary conditions (14) and (15) become $F_{1}=F_{3}=0, F_{1}^{\prime}=F_{3}^{\prime}=0, G_{1}=1, G_{3}=0$, $T_{1}=\frac{1}{E_{c}}, T_{3}=0, \psi_{0}^{\prime}=-t_{d} \psi_{0} T_{1}^{\prime}, \psi_{1}^{\prime}=L^{*} G_{1}-$ $B_{d} \psi_{0}\left({F_{1}^{\prime}}^{2}+{G_{1}^{\prime}}^{2}-N F_{1}^{\prime}\right)-t_{d} \psi_{0} T_{3}^{\prime} \quad$ at $\eta=0$ 
$F_{1}^{\prime} \rightarrow 0, F_{3}^{\prime} \rightarrow 0, G_{1} \rightarrow 0, G_{3} \rightarrow 0, T_{1} \rightarrow 0, T_{3} \rightarrow 0, \psi_{0} \rightarrow$ $1, \psi_{1} \rightarrow 0$ as $\eta \rightarrow \infty$

where $E_{c}=\frac{a^{2} \Omega^{2}}{c_{v}\left(T_{w}-T_{\infty}\right)}$ is the Eckert number and $L^{*}=$ $\frac{\beta \sigma a \Omega B_{0}}{\rho D\left(C_{1}\right)_{\infty}}$ is the electric parameter.

Since the solutions of non-linear coupled ordinary differential equations (23) to (33) under the boundary conditions (34)-(35) cannot be obtained in closed form therefore these equations are solved numerically with MATLAB's built- in solver bvp4c. From the process of numerical computation, the local skin friction, the Nusselt number and the Sherwood number, which are respectively proportional to $\mathrm{F}_{1}^{\prime \prime}(0), \mathrm{F}_{3}^{\prime \prime}(0), \mathrm{G}_{1}^{\prime}(0), \mathrm{G}_{3}^{\prime}(0)$; $\mathrm{T}_{1}^{\prime}(0), \mathrm{T}_{3}^{\prime}(0)$; and $\psi_{0}^{\prime}(0), \psi_{1}^{\prime}(0)$ are also worked out and their numerical values are presented in a tabular form.

\section{RESULTS AND DICUSSION}

Numerical calculations have been carried out for various values of the parameters $\mathrm{N}, P_{r}, S_{c}, t_{d}, B_{d}, E_{c}$ and $\mathrm{L}^{*}$, and these numerical results for concentration of the lighter component of the binary fluid mixture are plotted against $\eta$ for various values of above mentioned parameters and are displayed in Figures 1-7. It is observed from the figures that the concentration of the lighter component of the binary mixture is more at the plate and decreases exponentially as $\eta$ increases to 4.5 . Thereafter in the region $\eta>4.5$ no variation in $\psi_{1}$ is observed. The concentration of the lighter and rarer component of the binary mixture increases sharply near the plate with decrease in the values of all the parameters namely $\mathrm{N}, P_{r}, S_{c}, t_{d}, B_{d}, E_{c}$ and L*.

Thus we conclude that the separation of the binary mixture can be enhanced by decreasing (i) the strength of the applied magnetic field, (ii) electrical conductivity of the medium, and by increasing the rate of rotation of the sphere. The effect of demixing is confined in a boundary layer region near the surface of the sphere. The lighter component of the binary fluid mixture gets collected near the surface of the sphere whereas the heavier one is thrown away from it.

Taking into account the conclusion derived in this paper, gas separating instruments can be installed in big cities where harmful gases are present in very small quantities that can be sucked after separating them and thus pollutants can be removed.

In the present investigation the effect of weak magnetic field on separation has been studied. There is scope for the study of the effect of strong magnetic field on separation of binary fluid mixture.

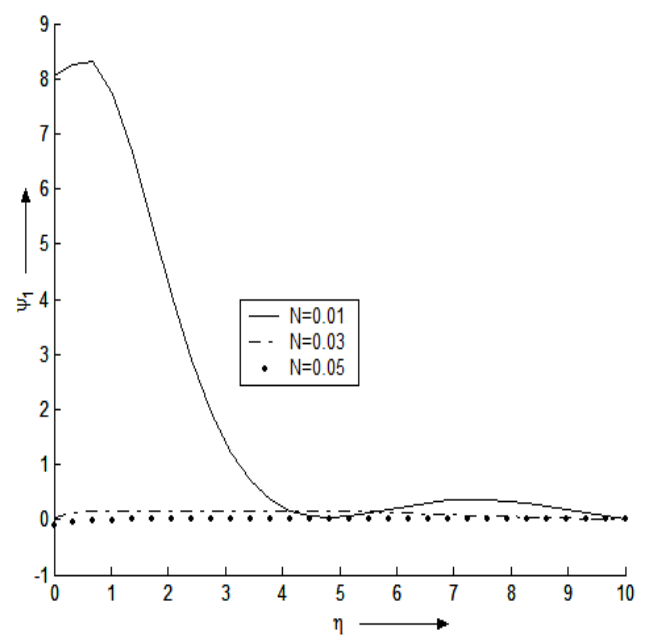

Figure.1. The graph of $\psi_{1}$ against $\eta P_{r}=1.1, S_{c}=$ $4, t_{d}=0.001, B_{d}=0.001, E_{c}=0.01$ and $L^{*}=4$ for various values of $\mathbf{N}$.

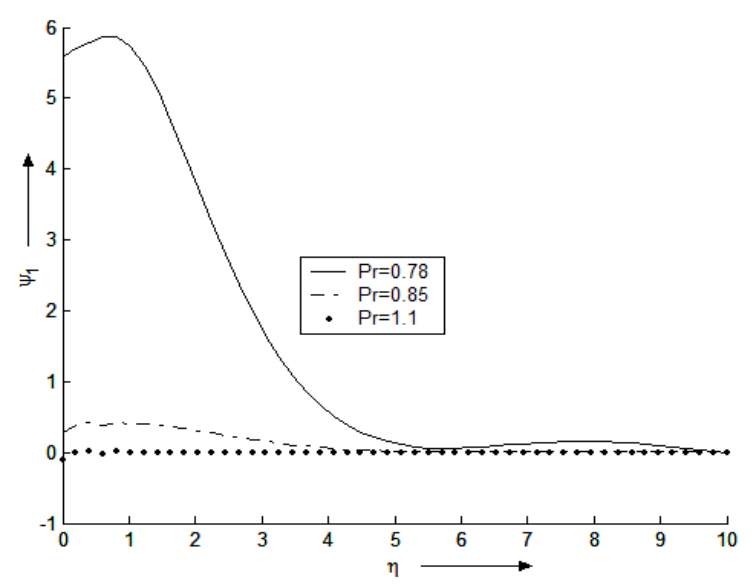

Figure.2. The graph of $\psi_{1}$ against $\eta \quad N=0.01$, $S_{c}=4, t_{d}=0.001, B_{d}=0.001, E_{c}=0.01$ and $L^{*}=4$ for various values of $P_{r}$.

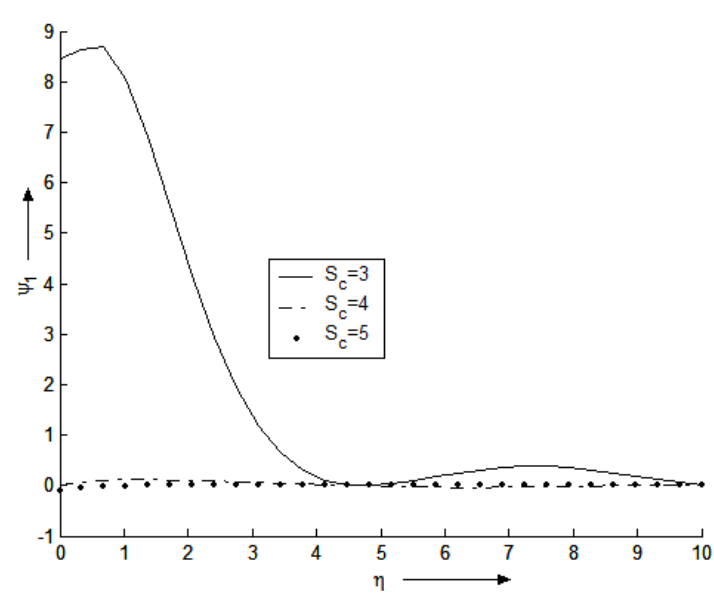

Figure.3. The graph of $\psi_{1}$ against $\eta \quad P_{r}=1.1$, $N=0.01, t_{d}=0.001, B_{d}=0.001, E_{c}=0.01$ and $L^{*}=4$ for various values of $S_{c}$. 


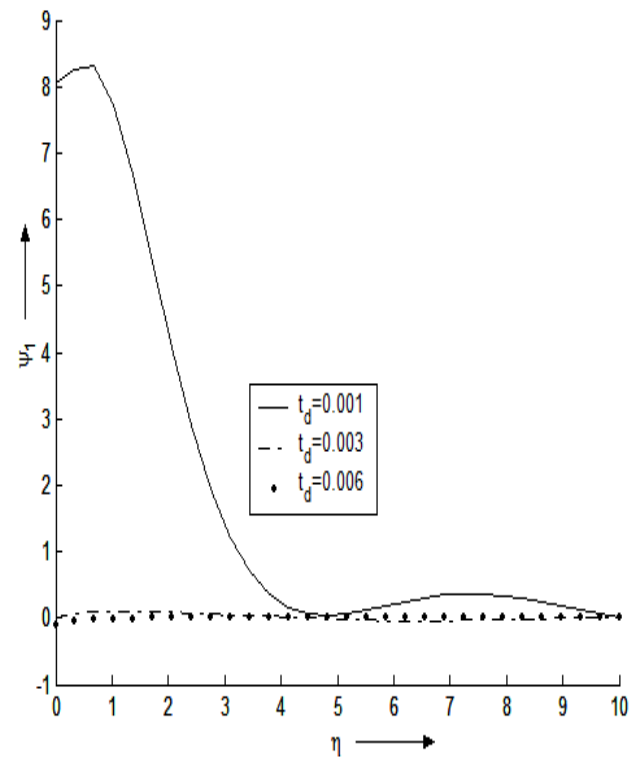

Figure.4. The graph of $\psi_{1}$ against $\eta \quad N=0.01$, $S_{c}=4, P_{r}=1.1, B_{d}=0.001, E_{c}=0.01$ and $L^{*}=4$ for various values of $t_{d}$.

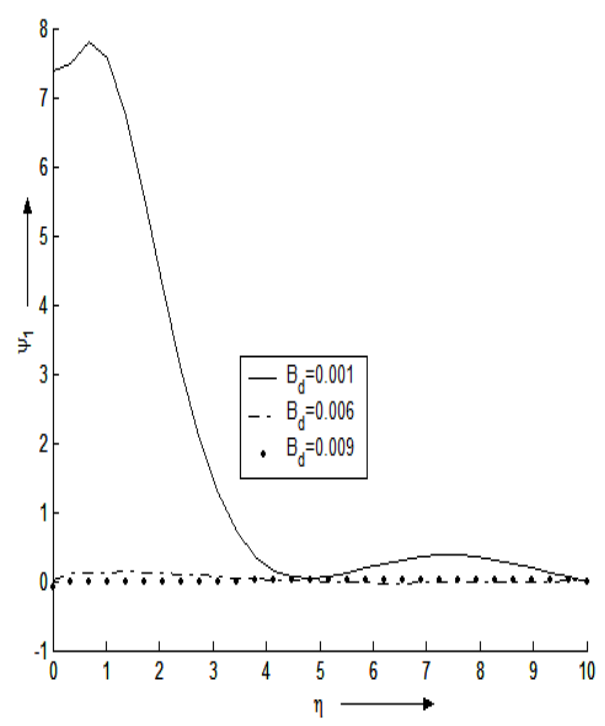

Figure.5. The graph of $\psi_{1}$ against $\eta$

$P_{r}=1.1, N=0.01, t_{d}=0.001, S_{c}=4$,

$E_{c}=0.01$ and $L^{*}=4$ for various values of $B_{d}$.

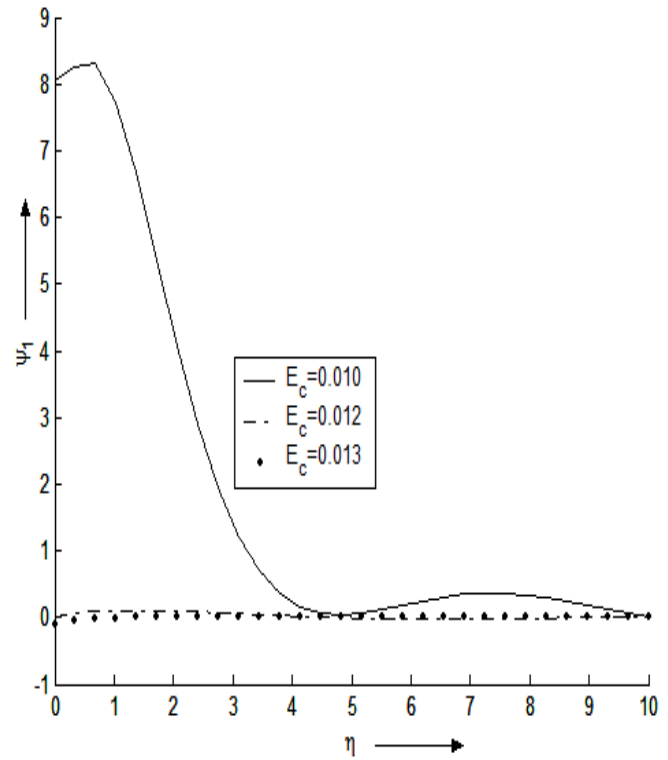

Figure.6. The graph of $\psi_{1}$ against $\eta \quad N=0.01$, $S_{c}=4, P_{r}=1.1, B_{d}=0.001, t_{d}=0.001$ and $L^{*}=4$ for various values of $E_{c}$.

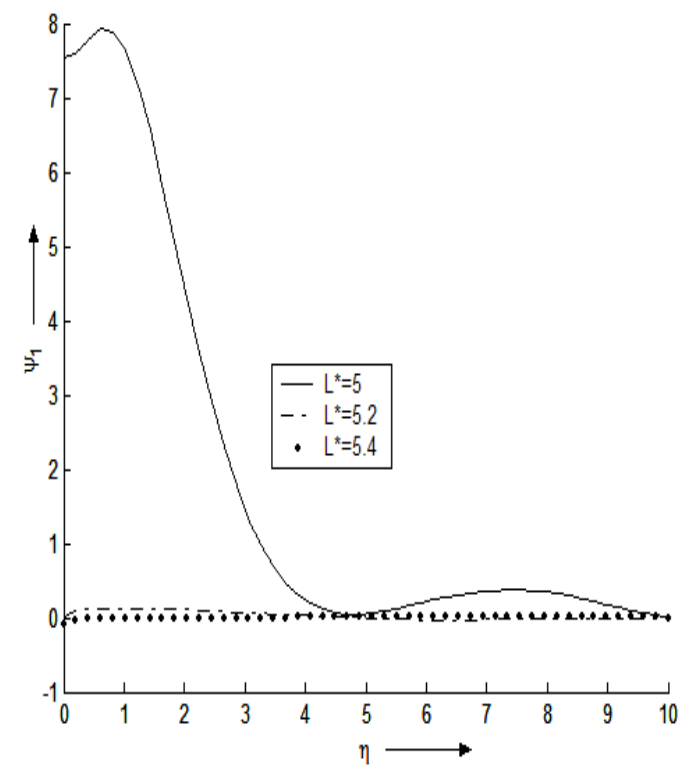

Figure.7. The graph of $\psi_{1}$ against $\eta$ $P_{r}=1.1, N=0.01, t_{d}=0.001, S_{c}=4$, $E_{c}=0.01$ and $B_{d}=0.001$ for various values of $L^{*}$.

Finally, the effects of the local skin friction, the Nusselt number and the Sherwood number are shown in Table1. The behaviour of these parameters is self - evident from the Table 1 and hence any further discussion about them seems to be redundant. 
Table1. Numerical values of $F_{1}, F_{1}^{\prime}, F_{1}^{\prime \prime}, F_{3}$, $F_{3}^{\prime}, F_{3}^{\prime \prime}, G_{1}, G_{1}^{\prime}, G_{3}, G_{3}^{\prime}, T_{1}, T_{1}^{\prime}, T_{3}, T_{3}^{\prime}, \psi_{0}, \psi_{0}^{\prime}, \psi_{1}$ and $\psi_{1}^{\prime}$ at $\eta=$ 0 .

\begin{tabular}{|c|c|c|c|c|c|c|c|c|c|}
\hline & \multicolumn{3}{|c|}{$\begin{array}{l}P_{r}=1.1, S_{c}=4, t_{d}= \\
0.001, B_{d}=0.001, \mathrm{E}_{\mathrm{c}}=0.01 \text { and } \\
\mathrm{L}^{*}=4\end{array}$} & \multicolumn{3}{|c|}{$\begin{array}{l}\mathrm{N}=0.01, S_{c}=4, t_{d}= \\
0.001, B_{d}=0.001, \mathrm{E}_{\mathrm{c}}=0.01 \text { and } \\
\mathrm{L}^{*}=4\end{array}$} & \multicolumn{3}{|c|}{$\begin{array}{l}P_{r}=1.1, N=0.01, t_{d}= \\
0.001, B_{d}=0.001, \mathrm{E}_{\mathrm{c}}=0.01 \text { and } \mathrm{L}^{*}=4\end{array}$} \\
\hline & \multicolumn{3}{|c|}{$\mathrm{N}$} & \multicolumn{3}{|c|}{$P_{r}$} & \multicolumn{3}{|c|}{$S_{c}$} \\
\hline & 0.01 & 0.03 & 0.05 & 0.78 & 0.85 & 1.1 & 3 & 4 & 5 \\
\hline$F_{1}$ & 0 & 0 & 0 & 0 & 0 & 0 & 0 & 0 & 0 \\
\hline$F_{1}^{\prime}$ & 0 & 0 & 0 & 0 & 0 & 0 & 0 & 0 & 0 \\
\hline$F_{1}^{\prime \prime}$ & 0.5206 & 0.4986 & 0.4950 & 0.5206 & 0.5119 & 0.5069 & 0.5206 & 0.5054 & 0.5071 \\
\hline$F_{3}$ & 0 & 0 & 0 & 0 & 0 & 0 & 0 & 0 & 0 \\
\hline$F_{3}^{\prime}$ & 0 & 0 & 0 & 0 & 0 & 0 & 0 & 0 & 0 \\
\hline$F_{3}^{\prime \prime}$ & 0.1264 & 0.0878 & 0.1143 & 0.1264 & 0.1133 & 0.1163 & 0.1264 & 0.1005 & 0.1179 \\
\hline$G_{1}$ & 1.0000 & 1.0000 & 1.0000 & 1.0000 & 1.0000 & 1.0000 & 1.0000 & 1.0000 & 1.0000 \\
\hline$G_{1}^{\prime}$ & -0.5732 & -0.6210 & -0.6389 & -0.5732 & -0.6020 & -0.6207 & -0.5732 & $\begin{array}{l}-0.6099 \\
\end{array}$ & -0.6205 \\
\hline$G_{3}$ & 0 & 0 & 0 & 0 & 0 & 0 & 0 & 0 & 0 \\
\hline$G_{3}^{\prime}$ & 0.1312 & 0.1164 & 0.1359 & 0.1312 & 0.1411 & 0.1366 & 0.1312 & 0.1308 & 0.1433 \\
\hline$T_{1}$ & 100 & 100 & 100 & 100 & 100 & 100 & 100 & 100 & 100 \\
\hline$T_{1}^{\prime}$ & -40.7245 & -39.5389 & -40.5560 & -32.1537 & -33.6062 & -41.4797 & -40.7245 & -39.8488 & -41.4977 \\
\hline$T_{3}$ & 0 & 0 & 0 & 0 & 0 & 0 & 0 & 0 & 0 \\
\hline$T_{3}^{\prime}$ & 6.5153 & 18.1229 & 8.1763 & 4.6503 & 10.5570 & 6.8934 & 6.5153 & 12.2907 & 8.5236 \\
\hline$\psi_{0}$ & 0.9710 & 0.9666 & 0.9648 & 0.9768 & 0.9723 & 0.9643 & 0.9706 & 0.9669 & 0.9661 \\
\hline$\psi_{0}^{\prime}$ & 0.0319 & 0.0382 & 0.0391 & 0.0254 & 0.0323 & 0.0400 & 0.0319 & 0.0385 & 0.0401 \\
\hline$\psi_{1}$ & 8.0588 & 0.0505 & -0.1071 & 5.5812 & 0.2719 & -0.1037 & 8.4553 & 0.0149 & -0.1048 \\
\hline$\psi_{1}^{\prime}$ & 3.9941 & 3.9815 & 3.9911 & 3.9955 & 3.9888 & 3.9924 & 3.9941 & 3.9871 & 3.9908 \\
\hline
\end{tabular}

\begin{tabular}{|c|c|c|c|c|c|c|c|c|c|c|c|c|}
\hline & \multirow{2}{*}{\multicolumn{3}{|c|}{$\begin{array}{lr}\mathrm{N}=\quad 0.01, & S_{c}=4, P_{r}= \\
1.1, B_{d}=0.001, & \mathrm{E}_{\mathrm{c}}= \\
0.01 \text { and } \mathrm{L}^{*}=4 & \\
& \\
& \\
t_{d} & \end{array}$}} & \multicolumn{3}{|c|}{$\begin{array}{l}P_{r}=1.1, \quad N=0.01, t_{d}= \\
0.001, S_{c}=4, \mathrm{E}_{\mathrm{c}}=0.01 \text { and } \\
\mathrm{L}^{*}=4\end{array}$} & \multicolumn{3}{|c|}{$\begin{array}{l}\mathrm{N}=\quad 0.01, \quad S_{c}=4, P_{r}= \\
1.1, B_{d}=0.001, \mathrm{t}_{\mathrm{d}}=0.001 \text { and } \\
\mathrm{L}^{*}=4\end{array}$} & \multicolumn{3}{|c|}{$\begin{array}{l}P_{r}=1.1, \quad N=0.01, t_{d}= \\
0.001, S_{c}=4, \\
\mathrm{E}_{\mathrm{c}}=0.01 \text { and } B_{d}=0.001\end{array}$} \\
\hline & & & & & $B_{d}$ & & & $\mathrm{E}_{\mathrm{c}}$ & & & $\mathrm{L}$ & \\
\hline & 0.001 & 0.003 & 0.006 & 0.001 & 0.006 & 0.009 & 0.010 & 0.012 & 0.013 & 5 & 5.2 & 5.4 \\
\hline$\overline{F_{1}}$ & 0 & 0 & 0 & 0 & 0 & 0 & 0 & 0 & 0 & 0 & 0 & 0 \\
\hline$F_{1}^{\prime}$ & 0 & 0 & 0 & 0 & 0 & 0 & 0 & 0 & 0 & 0 & 0 & 0 \\
\hline$F_{1}^{\prime \prime}$ & 0.520 & 0.505 & 0.507 & 0.520 & 0.505 & 0.507 & 0.520 & 0.505 & 0.507 & 0.520 & 0.505 & 0.507 \\
\hline$F_{3}$ & 0 & 0 & 0 & 0 & 0 & 0 & 0 & 0 & 0 & 0 & 0 & 0 \\
\hline$F_{3}^{\prime}$ & 0 & 0 & 0 & 0 & 0 & 0 & 0 & 0 & 0 & 0 & 0 & 0 \\
\hline$F_{3}^{\prime \prime}$ & 0.126 & 0.100 & 0.117 & 0.126 & 0.100 & 0.117 & 0.126 & 0.100 & 0.117 & 0.126 & 0.100 & 0.117 \\
\hline$G_{1}$ & 1.000 & 1.000 & 1.000 & 1.000 & 1.000 & 1.000 & 1.000 & 1.000 & 1.000 & 1.000 & 1.000 & 1.000 \\
\hline$G_{1}^{\prime}$ & -0.573 & -0.609 & -0.620 & -0.573 & -0.609 & -0.620 & -0.573 & -0.609 & -0.620 & -0.573 & -0.609 & -0.620 \\
\hline$G_{3}$ & 0 & 0 & 0 & 0 & 0 & 0 & 0 & 0 & 0 & 0 & 0 & 0 \\
\hline$G_{3}^{\prime}$ & 0.131 & 0.130 & 0.143 & 0.131 & 0.130 & 0.143 & 0.131 & 0.130 & 0.143 & 0.131 & 0.130 & 0.143 \\
\hline$T_{1}$ & 100 & 100 & 100 & 100 & 100 & 100 & 100 & 100 & 100 & 100 & 100 & 100 \\
\hline$T_{1}^{\prime}$ & -40.72 & -39.84 & -41.49 & -40.72 & -39.84 & -41.49 & -40.72 & -39.84 & -41.49 & -40.72 & -39.84 & $\begin{array}{l}-41.49 \\
\end{array}$ \\
\hline$T_{3}$ & 0 & 0 & 0 & 0 & 0 & 0 & 0 & 0 & 0 & 0 & 0 & 0 \\
\hline$T_{3}^{\prime}$ & 6.515 & 12.290 & 8.523 & 6.515 & 12.371 & 8.522 & 6.515 & 12.29 & 8.523 & 6.515 & 12.351 & 8.522 \\
\hline$\psi_{0}$ & 0.9710 & 1.000 & 1.049 & 0.971 & 0.966 & 0.964 & 0.971 & 0.966 & 0.964 & 0.971 & 0.966 & 0.964 \\
\hline$\psi_{0}^{\prime}$ & 0.031 & 0.039 & 0.043 & 0.031 & 0.038 & 0.040 & 0.031 & 0.038 & 0.040 & 0.031 & 0.038 & 0.040 \\
\hline$\psi_{1}$ & 8.058 & 0.010 & -0.106 & 7.381 & 0.012 & -0.104 & 8.058 & 0.011 & -0.105 & 7.549 & 0.012 & -0.104 \\
\hline$\psi_{1}^{\prime}$ & 3.994 & 3.986 & 3.990 & 3.994 & 3.987 & 3.990 & 3.994 & 3.987 & 3.990 & 3.994 & 3.987 & 3.990 \\
\hline
\end{tabular}




\section{ACKNOWLEDGEMENT}

This research work is funded by grants from the UGC, New Delhi, India (File No. 39-43/2010 (SR)) as a Major Research Project awarded to Dr. B. R. Sharma. Kabita Nath is associated with the project as a Project Fellow. The authors are grateful to UGC for providing financial support during the research work.

\section{REFERENCES}

[1] L.Howarth.1951.The boundary layer in three dimensional flow Part I. Phil. Mag. Ser. 7, 42, 239.

[2] S.D.Nigam.1954. Note in the boundary layer on a rotating sphere. Jour. Appl. Math. Phys. (ZAMP), 5, 151-155.

[3] W.H.H. Banks. 1965.The boundary layer on a rotating sphere. Quart. Jour. Mech. Appl. Maths, 18(4) , 443454 .

[4] S.N.Singh.1960. Heat transfer by laminar flow from a rotating sphere. Appl. Sci. Res., A9 , 197-205.

[5] W.H.H. Banks.1965. The thermal laminar boundary layer on a rotating sphere. Jour. Appl. Math. Phys. (ZAMP), 16(6) , 780-788.

[6] K.C. Kalita, Dibrugarh University Ph.D. Thesis 1982.

[7] V.C. Liu.1959. On the separation of gas mixtures by suction of the thermal diffusion boundary layer. Quart. Jour. Mech. Appl. Maths., 12(1), 1.

[8] Sharma BR, Singh RN. 2008. Barodiffusion and thermal diffusion a binary fluid mixture confined between two parallel discs in presence of a small axial magnetic field. Latin American Applied Research. ; 38: 313-320.
[9] Sharma BR, Singh RN.2009. Thermal diffusion in a binary fluid mixture confined between two concentric circular cylinders in presence of radial magnetic field. J. Energy Heat Mass Transfer. ; 31: 27-38.

[10] Sharma BR, Singh RN.2010. Separation of species of a binary fluid mixture confined between two concentric rotating circular cylinders in presence of a strong radial magnetic field. Heat Mass Transfer. 46: 769-777.

[11] B.R. Sharma, Kabita Nath.2012. The effect of magnetic field on separation of binary mixture of viscous fluids by barodiffusion and thermal diffusion near a stagnation point- a numerical study. Int. Jour. Mathematical Archive. 3(3), 1118-1124.

[12] Sharma BR , Singh RN, Gogoi Rupam Kr.2011. Effect of a Strong Transverse Magnetic Field on Separation of Species of a Binary Fluid Mixture in Generalized Couette Flow. Int. Journal of Applied Engineering Research. ; 6:2223-2235.

[13] B.R. Sharma, R.N Singh, Rupam Kr. Gogoi, Kabita Nath.2012. Separation of species of a binary fluid mixture confined in a channel in presence of a strong transverse magnetic field. Hem. Ind. 66(2), 171-180.

[14] Sharma BR, Singh RN.2004. Soret effect in generalized MHD Couette flow of a binary mixture. Bull Cal Math Soc. ; 96: 367-374.

[15] Sharma BR, Singh RN. 2007.Soret effect due to natural convection between heated vertical plates in a horizontal small magnetic field. Ultra Science. ; 19 97-106.

[16] L.D. Landau, E.M. Lifshitz.1959. Fluid Mechanics, $2^{\text {nd }}$ edn. London. Pergamen Press.

[17] D.T. Hurle, E. Jakeman.1971. Soret-driven thermosolutal convection, J. Fluid Mech. 47 , 667-687. 